\title{
Real-world efficacy, toxicity and clinical management of ipilimumab treatment in metastatic melanoma
}

\author{
LEILA KHOJA, ESHETU G. ATENAFU, QIAN YE, CRAIG GEDYE, MARYANNE CHAPPELL, \\ DAVID HOGG, MARCUS O. BUTLER and ANTHONY M. JOSHUA
}

Department of Medical Oncology, Princess Margaret Cancer Centre, Toronto, ON M5G 2M9, Canada

Received October 9, 2014; Accepted September 30, 2015

DOI: 10.3892/ol.2015.4069

\begin{abstract}
Approved by the Food and Drug Administration in 2011, the anti-cytotoxic T-lymphocyte-associated protein 4 checkpoint inhibitor ipilimumab has delivered a survival benefit of $\geq 3$ years in a subset of metastatic melanoma patients. After participating in the registration trial, patients were treated with this agent in routine practice. Toxicity and efficacy of agents in "real world" settings may differ from trials. The present study aimed to evaluate, with respect to toxicity and outcome, all patients treated with ipilimumab to date at the Princess Margaret Hospital (Toronto, Canada). Patients treated with ipilimumab between 2008 and 2013 were identified, and patient characteristics (age, gender, tumour burden, oncogenic mutation status, number of treatments received and toxicities from treatment) were collected. Progression-free survival (PFS) and overall survival (OS) were calculated from the commencement of ipilimumab treatment. Associations between clinical characteristics and outcome or toxicity were assessed. Between 2008 and 2013, 129 patients with metastatic cutaneous melanoma were treated. Since, during this period, ipilimumab was approved in the second line setting, ipilimumab was delivered in the second or subsequent line in all patients, and $70 \%$ did not receive any further anticancer therapy. Immune-related toxicities were observed, the onset of which varied from 1 to 162 days. The majority resolved within 6 weeks of the final treatment, with the exception of endocrinopathies and bowel related toxicity. The median PFS and OS were 2.83 and 8.44 months, respectively. No pre-treatment factor independently predicted toxicity. The number of infusions (4 vs. $\leq 3$ ) and presence of toxicity were significantly associated with superior survival. The onset of toxicity secondary to ipilimumab could occur later than previously reported. Toxicities were manageable, but required long-term vigilance.
\end{abstract}

Correspondence to: Dr Leila Khoja or Dr Anthony M. Joshua, Department of Medical Oncology, Princess Margaret Cancer Centre, 610 University Avenue, Toronto, ON M5G 2M9, Canada

E-mail: leila.khoja@uhn.ca

E-mail: anthony.joshua@uhn.ca

Key words: checkpoint inhibitors, melanoma, toxicity, ipilimumab, pembrolizumab, immune-related adverse events

\section{Introduction}

The incidence of cutaneous melanoma continues to rise in Canada. The estimated number of cases in Canada for 2014 is 6,500 , accounting for $\sim 3.5 \%$ of all cancer cases (1). The majority, up to $80 \%$ of cases, are cured by surgery alone. Until recently, the prognosis in cases that recurred has been poor, with a median overall survival (OS) time of 6 months in patients with metastatic disease. Molecularly targeted agents and immune checkpoint inhibitors have significantly altered this dismal prognosis (2).

Ipilimumab, an anti-cytotoxic T-lymphocyte-associated protein 4 (CTLA-4) antibody was the first agent to demonstrate a survival benefit in the treatment of metastatic cutaneous melanoma, initially as a second-line and later as a first-line treatment $(3,4)$. Toxicities or adverse events reported in these pivotal trials were immune-related adverse events (irAE), in keeping with the drug's mechanism of action. A subsequent meta-analysis of mature data from phase II and phase III trials, in addition to $>2,000$ patients treated on the international expanded access programme (EAP), has indicated a median OS time of 9.5 months, with durable responses beyond 3 years in $21 \%$ of patients, the majority of whom were treated outside a clinical trial population (5). Awareness and management of irAE have also improved since ipilimumab approval, and, whilst these side effects are manageable in the majority of patients, they may result in significant long-term morbidity and even, in rare cases, mortality $(3,4)$. The majority of irAE relate to cutaneous, hepatic or intestinal inflammation or endocrinopathies. However rarer neurological toxicity has also been documented in case reports (6). Newer checkpoint inhibitors targeting the programmed cell death 1 (PD-1)/programmed death-ligand 1 (PD-L1) axis are being evaluated in phase III trials, alone or in combination with ipilimumab. These agents appear to have greater activity and different patterns of immune toxicities $(7,8)$. Appreciation of potential irAE, when they may occur and their management will be crucial to treating patients as these agents become standard therapy, moving out of cancer centres and into community practice. Checkpoint inhibitors are additionally being evaluated as adjuvant treatments and early data has demonstrated a delay in disease recurrence. If these agents are approved in the adjuvant setting, a significantly higher number of patients will be eligible for treatment with these agents (9), as is the case now with the recent approval of adjuvant ipilimumab. 
The Princess Margaret Cancer Centre (Toronto, Canada) has the largest single centre experience of ipilimumab use in metastatic melanoma in Canada. The aim of the present study was to evaluate firstly the toxicity and secondly the outcomes of all patients treated at this institution from 2008 to date. Additionally, we sought to compare our practice with published literature and, drawing additionally on our own experience, to evaluate guidelines for the early detection and management of ipilimumab-related toxicity in the Canadian context.

\section{Patients and methods}

Patients. This retrospective review was conducted at the Princess Margaret cancer centre using a research ethics board-approved protocol and in accordance with the Declaration of Helsinki. Pharmacy records were searched to identify patients who had received ipilimumab between 2008 and 2013, inclusively. These patients' records were evaluated to collect data on gender, age, ECOG performance status, tumour burden, previous treatments, mutation status of primary tumours, number of ipilimumab infusions, response (by CT scan) at the end of treatment, toxicity (assessed by CTCAE, version 3) incurred during and following treatment, time to toxicity from the date of the first ipilimumab infusion, and survival outcomes, allowing calculation of progression free survival (PFS) and OS.

Statistical analysis. Associations between patient characteristics and toxicity) or survival were tested using univariate and then multivariate analysis (Chi square analysis and log rank test), using SAS version 9.2 (SAS, Cary, NC, USA). P<0.05 was considered to indicate a statistically significant difference.

\section{Results}

Patient profiles. A total of 129 patients with metastatic cutaneous melanoma were identified [for the purposes of this study, patients with uveal $(n=5)$, mucosal and acral melanomas $(n=24)$ were excluded]. All patients received ipilimumab as a second-line or higher treatment. All patients received $3 \mathrm{mg} / \mathrm{kg}$ infusions every 3 weeks up to a planned 4 cycles. In addition, 7 patients also received re-induction therapy, where a further 4 doses of ipilimumab were administered after previous clinical benefit from this treatment was demonstrated. Patient characteristics are shown in Table I, and all patients received $\geq 1$ ipilimumab infusion. The median and mean numbers of infusions received were 4 and 3 , respectively. Of the 129 patients, 9 had M1a, 11 had M1b and 109 had M1c disease. Examination of sites of metastases revealed that 33 patients $(25.58 \%)$ had bone metastases, $88(68.82 \%)$ had lung secondaries and $46(35.66 \%)$ had liver disease. In 112 patients $(86.82 \%)$, metastases were detected at other sites, including nodes, pancreas, spleen, adrenals or soft tissue masses. Brain metastases were present in 31 patients prior to commencing ipilimumab therapy, of which 13 subsequently progressed in the brain and required further radiotherapy. New brain metastases during or following ipilimumab treatment were detected in 18 patients.

Follow-up treatments. The majority of patients (n=91; 70.5\%) did not receive any active treatment following ipilimumab.
Table I. Patient characteristics $(\mathrm{n}=129)$.

\begin{tabular}{|c|c|}
\hline Characteristic & Value \\
\hline \multicolumn{2}{|l|}{ Age, years } \\
\hline Median & 57 \\
\hline Range & $24-83$ \\
\hline \multicolumn{2}{|l|}{ Gender, n } \\
\hline Female & 48 \\
\hline Male & 81 \\
\hline \multicolumn{2}{|l|}{ Performance status, $\mathrm{n}$} \\
\hline 0 & 33 \\
\hline 1 & 80 \\
\hline Unknown & 16 \\
\hline \multicolumn{2}{|l|}{ LDH (at baseline; U/l), $\mathrm{n}$} \\
\hline$\leq 220$ & 42 \\
\hline$>220$ & 83 \\
\hline Unknown & 4 \\
\hline \multicolumn{2}{|l|}{ AJCC M stage, $n$} \\
\hline M1a & 9 \\
\hline M1b & 11 \\
\hline M1c & 109 \\
\hline \multicolumn{2}{|l|}{ Ipilimumab - line of treatment, $\mathrm{n}$} \\
\hline Second line & 80 \\
\hline Third line & 36 \\
\hline Fourth line & 7 \\
\hline$\geq$ Fifth line & 6 \\
\hline \multicolumn{2}{|l|}{ Treatments prior to ipilimumab, $\mathrm{n}$} \\
\hline Chemotherapy & 105 \\
\hline Targeted agent/BRAF inhibitor & 29 \\
\hline Pembrolizumab (anti-PD1 antibody) & 7 \\
\hline High dose interleukin-2/other immunotherapy & 10 \\
\hline Trial (experimental agent) & 28 \\
\hline \multicolumn{2}{|l|}{$B R A F$ mutation status ${ }^{\mathrm{a}}, \mathrm{n}$} \\
\hline Positive & 33 \\
\hline Negative & 68 \\
\hline \multicolumn{2}{|l|}{$N R A S$ mutation status ${ }^{\mathrm{a}}, \mathrm{n}$} \\
\hline Positive & 13 \\
\hline Negative & 10 \\
\hline \multicolumn{2}{|l|}{ KIT mutation status ${ }^{\mathrm{a}}, \mathrm{n}$} \\
\hline Positive & 0 \\
\hline Negative & 5 \\
\hline
\end{tabular}

${ }^{a}$ Where tested. LDH, lactate dehydrogenase; AJCC, American Joint Committee on Cancer staging system; PD1, programmed cell death 1; NRAS, neuroblastoma RAS oncogene.

Of those that were subsequently treated, $25(19.4 \%)$ received one further line of treatment, $9(7 \%)$ received two and $4(3.1 \%)$ received more than two further lines of treatment. These treatments included chemotherapy, BRAF inhibitor or other targeted agent, re-induction with ipilimumab, another immunotherapy (including anti-PD-1 antibodies), adoptive cell transfer of lymphocytes with interleukin 2 , or treatment on a 


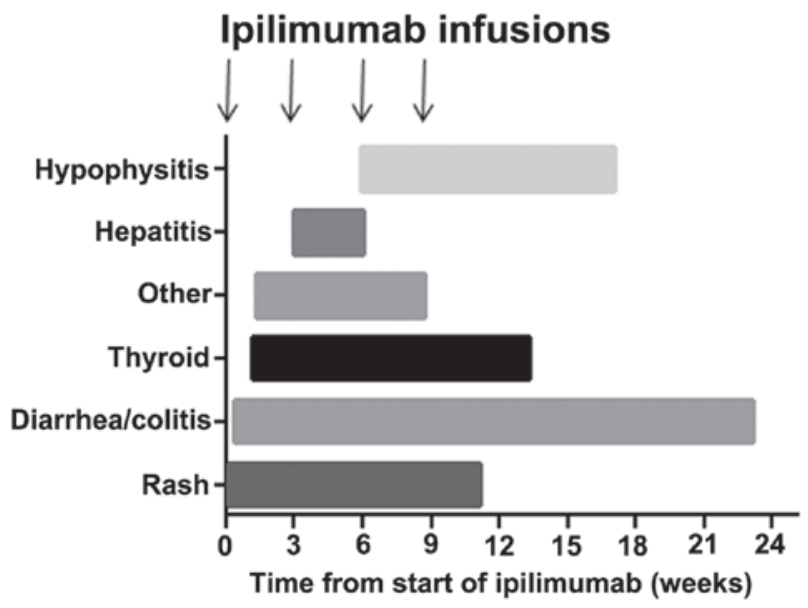

Figure 1. Bar chart depicting the onset of toxicity. Toxicities grouped according to immune-related adverse events relative to start of ipilimumab treatment.

clinical trial with an experimental targeted therapy or chemotherapy agent. Notably, 7 patients received anti-PD-1 antibody followed by ipilimumab treatment (of which 5 had progressive disease, 1 had stable disease and 1 had a partial response), whilst 10 patients received ipilimumab followed by anti-PD-1 antibody (of which 6 had progressive disease, 2 stable disease and 2 a partial response).

Toxicities. Treatment related toxicities included thyroid dysfunction, diarrhoea and autoimmune colitis, hepatitis, hypophysitis, rash or other autoimmune toxicities. All toxicities are described in Table II, in addition to the median time to onset, treatment thereof and outcome from treatment. Fig. 1 shows the onset of toxicity in the current sample of patients relative to the commencement of ipilimumab treatment. In 79 patients $(61.2 \%)$, no toxicities were experienced during or following treatment, whilst 27 patients (20.9\%) experienced one toxicity, 20 patients $(15.5 \%)$ experienced two, and 3 patients (2\%) experienced three toxicities. The majority of toxicities resolved, with the exception of endocrinopathies (hypophysitis or thyroid dysfunction) and bowel toxicity, which required long-term treatment or, in the case of autoimmune colitis, surgery in two patients. Colitis and endocrinopathies were observed to occur even after treatment had been completed, in contrast to other toxicities which occurred within the 12-week period of treatment and resolved within 6 weeks of the final dose of ipilimumab. Glucocorticoids were used in a total of 34 (26\%) patients to treat diarrhoea, hepatitis, rash, hypophysitis, arthritis, myositis or suspected pneumonitis. The median time to steroid treatment was 6.6 weeks (range, 2.7-27.3 weeks). Infliximab was required in 7 patients for refractory diarrhoea despite steroids; 2 of these patients required repeated infusions, 1 of whom subsequently underwent a resection with ileostomy formation. The median time to infliximab treatment was 7.6 weeks (range, 4.7-34.7 weeks). Univariate analysis indicated bone metastases $(\mathrm{P}=0.0373)$ to be associated with toxicity, whilst NRAS mutation appeared to be associated with reduced toxicity $(\mathrm{P}=0.024)$. Notably, the number of ipilimumab infusions received and the number of previous treatments were not significantly associated with
A

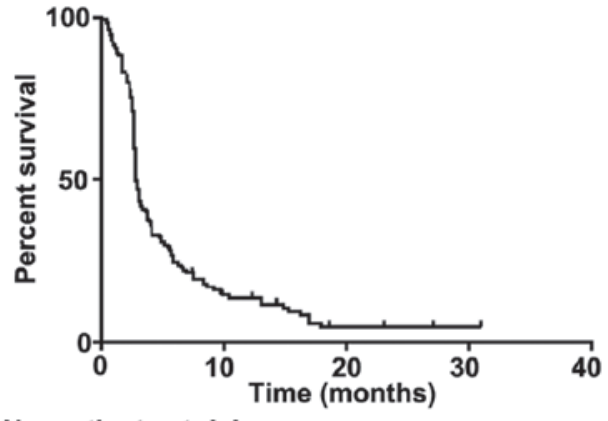

No. patients at risk

129

B

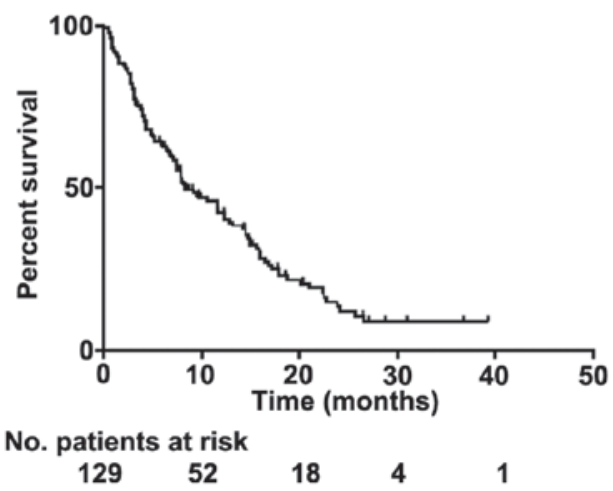

Figure 2. Kaplan-Meier curves demonstrating patient survival. (A) Progression-free survival for all patients; (B) overall survival for all patients.

toxicity. No factors were independently significantly associated with toxicity on multivariate analysis.

Response to treatment and survival outcomes. Response was determined by CT scan 4 weeks after the fourth cycle of ipilimumab. If stable disease or a response was determined then a confirmatory scan was performed 4 weeks thereafter, and patients followed up every three months with scans unless the clinical picture dictated otherwise. Of the 129 treated patients, 90 (70\%) had progressive disease and 37 (28\%) had either a partial response $(n=21$, one of whom subsequently achieved a complete response) or stable disease $(n=16)$. In 2 patients who were lost to follow-up, the response was unknown. Only 1 patient with progressive disease at the end of treatment went on to have a delayed response, which occurred 6 months later, and 1 patient with stable disease exhibited disease progression at a solitary site, which was resected with no further evidence of disease progression to date. Univariate analysis indicated that the factors positively associated with a response were the number of infusions received (4 infusions was superior to $\leq 3 \mathrm{P}=0.0027$ ), better baseline performance status ( 0 vs. 1 , $\mathrm{P}=0.0247), N R A S$ mutation $(\mathrm{P}=0.0022)$ and development of any toxicity $(\mathrm{P}=0.0299)$. By contrast, patients with bone metastases were less likely to respond to treatment $(\mathrm{P}=0.0166)$. No significant effect was associated with line of treatment, $B R A F$ mutation, gender or age. Multivariate analysis revealed $<4$ infusions $(\mathrm{P}=0.003)$ and male gender were associated with progressive disease $(\mathrm{P}=0.0484)$. The median follow-up time was 8 months (range, 1-39 months). At the time of analysis, 101 patients were deceased and 28 were alive. The median 
Table II. Toxicities during ipilimumab treatment, management thereof and outcome in 129 patients.

\begin{tabular}{|c|c|c|c|c|}
\hline Toxicity & $\begin{array}{l}\text { Patients, } \\
\mathrm{n}(\%)\end{array}$ & $\begin{array}{l}\text { Time to irAE onset, } \\
\text { days; median (range) }\end{array}$ & Treatment & Resolution of toxicity \\
\hline Diarrhoea & $34(26.4)$ & $36(3-162)$ & $\begin{array}{l}\text { Steroids used for grade } \geq 2 \text { in } \\
19 \text { patients; inflixamab in } 7 \\
\text { of these; Surgery required in } \\
2 \text { patients [total of } 8 \text { patients } \\
(6 \%) \text { with severe colitis] }\end{array}$ & $\begin{array}{l}\text { Responded to treatment apart from } \\
\text { in } 1 \text { patient who required ileostomy; } \\
1 \text { patient had a colectomy but failed } \\
\text { to present beforehand with symptoms } \\
\text { to allow medical treatment }\end{array}$ \\
\hline Rash & $18(14.0)$ & $20(1-78)$ & $\begin{array}{l}\text { Prednisone required in } 7 \\
\text { patients for grade } \geq 2 \text { rash }\end{array}$ & $\begin{array}{l}\text { Resolved completely post ipilimumab } \\
\text { treatment }\end{array}$ \\
\hline Thyroid dysfunction & $8(6.2)$ & $33(8-93)$ & $\begin{array}{l}\text { Thyroxine required in } 5 \\
\text { patients }\end{array}$ & $\begin{array}{l}\text { Thyroxine continued post ipilimumab } \\
\text { in all cases }\end{array}$ \\
\hline Hypophysitis & $6(4.7)$ & $63(41-119)$ & $\begin{array}{l}\text { Steroid replacement in all } 6 \\
\text { patients }\end{array}$ & $\begin{array}{l}\text { Steroid replacement needed long-term } \\
\text { post ipilimumab treatment }\end{array}$ \\
\hline Hepatitis & $4(3.1)$ & $35(21-42)$ & $\begin{array}{l}\text { Prednisone used in } 2 \text { patients } \\
\text { for grade } 3 \text { hepatitis }\end{array}$ & Completely resolved \\
\hline Other toxicity & $6(4.7)$ & $41(9-61)$ & $\begin{array}{l}\text { Encephalopathy, prednisone } \\
\text { given, resolved within } 24 \text { h } \\
\text { episcleritis, no treatment; } \\
\text { myositis, treated with } \\
\text { prednisone; arthritis, treated } \\
\text { with prednisone; pulmonary } \\
\text { inflammation, treated with } \\
\text { prednisone. }\end{array}$ & $\begin{array}{l}\text { All toxicities completely resolved } \\
\text { within } 6 \text { weeks from the last } \\
\text { ipilimumab treatment }\end{array}$ \\
\hline
\end{tabular}

irAE, immune-related adverse event.

PFS time was 2.83 months [95\% confidence interval (CI), 2.76-3.32 months] and the median OS time was 8.44 months (95\% CI, 7.13-12.42 months) (Fig. 2). The 1- and 2-year overall survival rates were $42 \%$ and $13 \%$, respectively, whilst survival for $\geq 3$ years was observed in $8.6 \%$ of patients.

Factors associated with superior PFS by univariate analysis, were low lactate dehydrogenase (LDH) level $(<220 \mathrm{u} / \mathrm{l}$; $\mathrm{P}<0.0001$ ), number of infusions (4 vs. $\leq 3, \mathrm{P}=0.0002$ ), toxicity during treatment $(\mathrm{P}=0.0061)$ and female gender $(\mathrm{P}=0.0407)$. Patients with liver $(\mathrm{P}=0.0006)$ or bone metastases $(\mathrm{P}=0.0092)$ had poorer PFS. Similar characteristics were significantly associated with superior OS: Low LDH $(\mathrm{P}<0.001)$, number of infusions $(\mathrm{P}<0.0001)$, NRAS mutation $(\mathrm{P}=0.0241)$ and female gender $(\mathrm{P}=0.046)$. Bone metastases and liver metastases were associated with poorer $\mathrm{OS}(\mathrm{P}=0.0004$ and $\mathrm{P}=0.0015$, respectively). Multivariate analysis indicated that $<4$ infusions $(\mathrm{P}<0.0001$ and $\mathrm{P}<0.0001)$ and absence of toxicity $(\mathrm{P}=0.0002$ and $\mathrm{P}=0.033$ ) were prognostic factors for poor PFS and OS, respectively; the presence of liver metastases was a significant factor for inferior OS only ( $\mathrm{P}=0.0048)$.

\section{Discussion}

The current study reports the real-world efficacy and toxicity of the novel anti-CTLA-4 antibody ipilimumab in patients treated primarily outside of clinical trials. The toxicities reported in the current sample of patients were irAE, in accordance with the published data on ipilimumab toxicity (6). These were, as expected, primarily related to dermatological, endocrine or gastrointestinal complications. In addition, a number of rarer toxicities, also assumed to be irAE, were observed (Table II).

A previous comprehensive review of 14 phase II-III ipilimumab trials in metastatic melanoma determined the most frequent grade $\geq 3$ toxicities, in a pooled analysis of 1,498 patients, to be diarrhoea (7\% of patients), colitis $(4.9 \%)$, perforation $(0.3 \%)$, rash (2.5\%), any liver dysfunction (2.1\%), hypothyroidism $(0.1 \%)$ and hypopituitarism (2.1\%) (6). Gastrointestinal toxicity of any grade occurred in $33 \%$ of patients, and 3 succumbed to complications associated with grade 3 or above gastrointestinal toxicity. Dermatological toxicity accounted for the majority of irAEs, with $65 \%$ of patients reporting some grade of rash; however, $<3 \%$ had grade $\geq 3$. Hepatic toxicity was more rare, occurring in $<2 \%$ of patients, and with $1 \%$ experiencing grade $3-4$. The endocrinopathies reported were related to thyroid dysfunction, hypopituitarism/hypophysitis or adrenal insufficiency, with an overall incidence of $<5 \%$ and a grade $\geq 3$ incidence of $<3 \%$ across all phase II-III trials. The time to onset of toxicity varied according to the irAE; rash was the earliest toxicity, followed by diarrhoea or colitis, then hepatic toxicity, and finally hypophysitis. The majority of toxicities appeared and resolved within the 12-week period (4 cycles) of ipilimumab treatment, with the exception of hypophysitis and liver toxicity, which in some cases could take longer to resolve or, in the case of hypophysitis, fail to resolve completely (6). In contrast to the reviewed data, the present study demonstrates that all of these toxicities have a wider range of times to onset and may occur much earlier in the course of treatment (Table II and Fig. 1). One major difference to the pooled data review described was the onset of diarrhoea; this 
appeared to commence much later in some of the current patients and extended well beyond the period of ipilimumab treatment.

There are no validated biomarkers for the prediction of toxicity, response or outcome to ipilimumab. The current data set is small and thus any associations are to be treated with caution. Nevertheless, others have reported that response is associated with NRAS mutation or autoimmune toxicity $(10,11)$. High $\mathrm{LDH}$ level is known to be a poor prognostic factor in metastatic melanoma, and has been suggested as a selection criterion for ipilimumab treatment (12). It most likely reflects overall disease status in addition to performance status. The survival outcomes in the present study are similar to those reported by other groups, reflecting the poorer clinical status of patients treated outside trials and particularly in the SAP and EAP, where ipilimumab may have been used as a higher line of therapy $(11,12)$.

The management of irAE is dependent upon vigilance in patient monitoring and early detection. If detected early, toxicities may be prevented from escalating, particularly in the case of gastrointestinal, hepatic and dermatological irAE. A high index of suspicion is required for the early detection of endocrinopathies, as early symptoms are often non-specific. It is unclear whether early immunosuppressive therapy can abrogate or mitigate the extent of endocrinopathies once these begin. It is essential that patients are made aware of the potential side effects and the need to communicate any symptoms to their clinical team. The average onset of different toxicities may guide physicians; however, given the variability between patients in the time to onset of toxicities (of any kind), this cannot be relied upon. Extensive guidelines for the management of ipilimumab-related toxicity are available as part of the Food and Drug Administration (FDA)-approved YERVOY ${ }^{\circledR}$ (ipilimumab) Risk Evaluation and Mitigation Strategy (REMS) (https://www.hcp. yervoy.com/pages/rems.aspx). These have been expanded further for greater guidance (6). The REMS also includes a checklist of symptoms and actions for healthcare workers to assess patients in community clinics or emergency departments, and a patient wallet card explaining potential irAEs and reminding patients to seek urgent medical attention for specific irAEs. These are useful adjuncts, particularly for general medical staff or emergency departments where many patients will present. Our current practice mandates a patient teaching session as to potential irAEs prior to commencing ipilimumab. Patients are seen prior to every cycle of treatment and every three months thereafter (depending on the course of treatment), undergoing full thyroid function tests (triiodothyronine, thyroxine and thyroid-stimulating hormone) and random cortisol and adrenocorticotropic hormone (and testosterone) evaluations, in addition to routine biochemical and haematological blood tests.

Pembrolizumab, the anti-PD-1 antibody inhibitor, was granted FDA approval in September 2014. In contrast to ipilimumab, newer checkpoint inhibitors that target the PD-1/PD-L1 axis have a different toxicity profile. Gastrointestinal toxicity and hypophysitis are less common, whilst pneumonitis occurs in $\sim 1 \%$ and may be treatment limiting (8). The kinetics of toxicity with these agents has not been fully defined and, unlike ipilimumab where treatment consists of 4 cycles only, these agents may be continued for up to 2 years. Our experience with anti-PD-1 agents indicates that thyroid dysfunction is common and early, detectable within the first 6 weeks. It tends firstly to hyperthyroidism, which is rarely symptomatic, and then to euthyroidism or hypothyroidism, requiring treatment. All other toxicities appear to occur at any time during treatment and, in certain cases, a year after the initiation of treatment (unpublished data).

In conclusion, there is little doubt that antibodies like ipilimumab that act as immune checkpoint inhibitors have significantly altered treatment paradigms in metastatic melanoma (2). It is likely that greater toxicity will be reported as these agents are more widely used; however, certain irAEs, particularly neurological toxicities, will be rare and idiosyncratic. Careful observation and early intervention via a multidisciplinary approach will therefore be required to optimally manage these patients. In addition the use of checkpoint inhibitors is likely to expand to other tumour types, and it will be necessary for non-oncology physicians to be familiar with their potential toxicities and their management.

\section{Acknowledgements}

Dr Leila Khoja was supported by grants from the Canadian Institutes of Health Research and the Guiletti Family Fellowship fund. Dr Craig Gedye was supported by a Canadian Institutes of Health Research/Kidney Cancer Canada Fellowship, a Royal Australasian College of Physicians CSL Fellowship, is supported by a National Health and Medical Research Council Overseas Postdoctoral Fellowship, and is a Hunter Cancer Research Alliance Clinical Research Fellow.

\section{References}

1. Canadian Cancer Society: Canadian Cancer Statistics.http://www. cancer.ca/en/cancer-information/cancer-101/canadian-cancer-stat istics-publication/?region=sk. Accessed 1 August 2014.

2. Gedye C, Hogg D, Butler M and Joshua AM: New treatments for metastatic melanoma. CMAJ 186: 754-760, 2014.

3. Hodi FS, O'Day SJ, McDermott DF, et al: Improved survival with ipilimumab in patients with metastatic melanoma. N Engl J Med 363: 711-723, 2010.

4. Robert C, Thomas L, Bondarenko I, et al: Ipilimumab plus dacarbazine for previously untreated metastatic melanoma. N Engl J Med 364: 2517-2526, 2011.

5. Schadendorf D, Robert C, Weber JS, et al: Pooled analysis of long-term survival data from phase II and phase III trials of ipilimumab in metastatic or locally advanced, unresectable melanoma. J Clin Oncol 33: 1889-1894, 2015.

6. Tarhini A: Immune-mediated adverse events associated with ipilimumab ctla-4 blockade therapy: The underlying mechanisms and clinical management. Scientifica (Cairo) 2013: 857519, 2013.

7. Topalian SL, Sznol M, McDermott DF, et al: Survival, durable tumor remission, and long-term safety in patients with advanced melanoma receiving nivolumab. J Clin Oncol 32: 1020-1030, 2014.

8. Robert C, Ribas A, Wolchok JD, et al: Anti-programmed-death-re ceptor-1 treatment with pembrolizumab in ipilimumab-refractory advanced melanoma: A randomised dose-comparison cohort of a phase 1 trial. Lancet 384: 1109-1117, 2014.

9. Eggermont AM, Chiarion-Sileni V, Grob JJ, et al: Ipilimumab versus placebo after complete resection of stage III melanoma: Initial efficacy and safety results from the EORTC 18071 phase III trial. ASCO Meeting Abstracts 32: LBA9008, 2014.

10. Johnson DB, Lovly CM, Flavin M, et al: NRAS mutation: A potential biomarker of clinical response to immune-based therapies in metastatic melanoma (MM). ASCO Meeting Abstracts 31: 9019, 2013.

11. Zimmerman ZF, Storer B, Godara A, et al: Outcomes and clinical markers associated with benefit from ipilimumab (Ipi) in patients with advanced melanoma: A retrospective single-institution study. ASCO Meeting Abstracts 31: e20048, 2013.

12. Kelderman S, Heemskerk B, van Tinteren H, et al: Lactate dehydrogenase as a selection criterion for ipilimumab treatment in metastatic melanoma. Cancer Immunol Immunother 63: 449-458, 2014. 